Meta

Journal des traducteurs

Translators' Journal

\title{
Quand Furetière menait la danse
}

\section{Le vocabulaire de la danse et les trois grands dictionnaires du $\mathrm{XVII}^{\mathrm{e}}$ siècle}

\section{Eugénia Roucher-Kougioumtzoglou}

Volume 39, numéro 4, décembre 1994

Hommage à Bernard Quemada : termes et textes

URI : https://id.erudit.org/iderudit/003996ar

DOI : https://doi.org/10.7202/003996ar

Aller au sommaire du numéro

Éditeur(s)

Les Presses de l'Université de Montréal

ISSN

0026-0452 (imprimé)

1492-1421 (numérique)

Découvrir la revue

\section{Citer cet article}

Roucher-Kougioumtzoglou, E. (1994). Quand Furetière menait la danse : le vocabulaire de la danse et les trois grands dictionnaires du XVII ${ }^{\mathrm{e}}$ siècle. Meta, 39(4), 716-730. https://doi.org/10.7202/003996ar

\section{Résumé de l'article}

Le vocabulaire de la danse classique, descendante directe de la belle danse du $\mathrm{XVII}^{\mathrm{e}}$ siècle, est en français dans le monde entier. Il fut codifié entre 1660 et 1680 et diffusé au moyen de la Chorégraphie, système de notation du mouvement mis au point en 1700 permettant de consigner par écrit la totalité du vocabulaire de la danse. Le but de cet article est de montrer la place non négligeable qu'occupé ce vocabulaire dans les trois grands dictionnaires de la fin du XVII ${ }^{\mathrm{e}}$ siècle -Dictionnaire François , Dictionnaire Universel, et Dictionnaire de l'Académie avec son supplément technique, le Dictionnaire des Arts et des Sciences de Thomas Corneille. L'étude est composée de trois chapitres. Le premier présente un rapide tour d'horizon de la place accordée aux vocabulaires techniques à l'intérieur de la production lexicographique française en fonction des options choisies par chacun des dictionnaires étudiés. Dans le deuxième, on procède à une comparaison entre eux portant sur deux points : a) la nomenclature relative à la danse ; b) le traitement du matériau lexical notamment en ce qui concerne l'organisation des articles, les définitions ainsi que l'utilisation des exemples, des citations et des collocations. Le troisième chapitre, enfin, démontre la supériorité de Furetière tant pour le linguiste que pour le choréologue. En effet, la richesse de sa nomenclature, l'enregistrement par son auteur d'un certain nombre de termes bien avant leur parution au XVIII ${ }^{\mathrm{e}}$ siècle dans des ouvrages spécialisés, ainsi qu'un travail définitionnel complet et précis, font du Dictionnaire Universel une source première inestimable non seulement pour l'étude du vocabulaire de la danse mais aussi pour la reconstitution fidèle des danses de l'époque.
Tous droits réservés ㄷ Les Presses de l'Université de Montréal, 1994
Ce document est protégé par la loi sur le droit d'auteur. L’utilisation des services d’Érudit (y compris la reproduction) est assujettie à sa politique d'utilisation que vous pouvez consulter en ligne.

https://apropos.erudit.org/fr/usagers/politique-dutilisation/ 


\title{
QUAND FURETIÈRE MENAIT LA DANSE LE VOCABULAIRE DE LA DANSE ET LES TROIS GRANDS DICTIONNAIRES DU XVIIE SIĖCLE
}

\author{
EUGÉNIA ROUCHER-KOUGIOUMTZOGLOU \\ Lycée Victor Duruy, Paris, France
}

\begin{abstract}
Résumé
Le vocabulaire de la danse classique, descendante directe de la belle danse du XVII siècle, est en français dans le monde entier. Il fut codifié entre 1660 et 1680 et diffusé au moyen de la Choregraphie, système de notation du mouvement mis au point en 1700 permettant de consigner par écrit la totalité du vocabulaire de la danse.

Le but de cet article est de montrer la place non négligeable qu'occupe ce vocabulaire dans les trois grands dictionnaires de la fin du XVIIe siècle - Dictionnaire François, Dictionnaire Universel, et Dictionnaire de l'Académie avec son supplément technique, le Dictionnaire des Arts et des Sciences de Thomas Corneille.

L'étude est composée de trois chapitres. Le premier présente un rapide tour d' horizon de la place accordée aux vocabulaires techniques à l'intérieur de la production lexicographique française en fonction des options choisies par chacun des dictionnaires étudiés. Dans le deuxième, on procède à une comparaison entre eux portant sur deux points : a) la nomenclature relative à la danse; b) le traitement du matériau lexical notamment en ce qui concerne l'organisation des articles, les définitions ainsi que l'utilisation des exemples, des citations et des collocations. Le troisième chapitre, enfin, démontre la supériorité de Furetière tant pour le linguiste que pour le choréologue. En effet, la richesse de sa nomenclature, l'enregistrement par son auteur d'un certain nombre de termes bien avant leur parution au XVIIIe siècle dans des ouvrages spécialisés, ainsi qu' un travail définitionnel complet et précis, font $d u$ Dictionnaire Universel une source première inestimable non seulement pour l'étude du vocabulaire de la danse mais aussi pour la reconstitution fidèle des danses de l'époque.
\end{abstract}

\begin{abstract}
The vocabulary of classical dance, a direct descendant of the belle danse of the seventeenth century, is in French the whole world over. It was codified between 1660 and 1680 and made known by La Choréographie, a system of notation of movements elaborated in 1700, making it possible to commit to paper all of dance vocabulary. The aim of this article is to show how important this vocabulary was in the three great dictionaries of the end of the seventeenth century: Dictionnaire François, Dictionnaire universel, and Dictionnaire de l'Academie, with its technical supplement by Thomas Corneille. The study is divided into three parts. The first is an overview of the importance given to technical vocabularies as a whole in French lexicography and with regard to the options chosen for each of the dictionaries. The second is a comparison of the dictionaries on two points: a) their nomenclature of dance terms; b) the treatment of lexical material especially with regard to the organisation of the articles, the definitions, the use of examples, quotations and collocations. The final part shows how superior Furetière was both for the linguist and the choreolog. The richness of his nomenclature, his recording of a certain number of terms well before their attestations in eighteenth century specialized works, and a complete and precise work on the definitions, all go to making the Dictionnaire universel a primary source of inestimable quality not only for the study of dance vocabulary but also for an accurate reconstitution of the dances of the period.
\end{abstract}


La danse, un des trois exercices principaux de la Noblesse, avec l'escrime et l'équitation, connut une vogue sans précédent en France sous le règne des Valois et des Bourbons. Née dans les Cours italiennes du Quattrocento, implantée et enracinée en France, la Belle Dance, ancêtre de la danse classique actuelle, est alors pratiquée par les Seigneurs et les Dames de la Cour. La fondation, en 1661, de l'Académie Royale de Danse par Lettres Patentes de Louis XIV, permit l'essor de cet art que cette Institution était chargée «d'augmenter et de perfectionner». Enfin, la création en 1669 des «Academies d'Opera», lui offrit le cadre sinon exclusif du moins définitif à l'intérieur duquel il évolua vers plus de professionalisme. Cette intervention du Pouvoir eut comme conséquence la codification du vocabulaire dont, en outre, l'invention par Feuillet de la Choregraphiel - écriture de la danse, au sens étymologique du terme - favorisa la diffusion dans toutes les Cours d'Europe à partir de 1700 . Depuis cette époque le vocabulaire de la danse classique est en français dans le monde entier.

Le propre de cet art ayant toujours été la prédominance du geste sur la parole et de celle-ci sur l'écriture, les documents écrits dont dispose le chercheur pour étudier l'activité chorégraphique du XVII ${ }^{\mathrm{e}}$ siècle et le vocabulaire qui s'y rattache, sont relativement peu nombreux. Le recours aux dictionnaires pour combler les lacunes s'avère donc plus qu'indispensable. Encore faut-il pour que cette consultation soit fructueuse, une connaissance du produit dictionnaire, de ses objectifs, des choix lexicographiques opérés par ses auteurs et des normes dictionnairiques auxquelles il est soumis.

L'objectif de cet article est d'examiner la façon dont les trois grands dictionnaires de la fin du siècle traitent le vocabulaire de la danse, d'évaluer cette nomenclature, de considérer la part qui revient au vocabulaire technique et celle qui concerne sa fraction banalisée; en un mot, d'essayer de dégager l'image qu'ils donnent de la danse en comparant et en rééquilibrant les renseignements fournis par eux plutôt qu'en les additionnant.

Le corpus est constitué essentiellement du Dictionnaire François ${ }^{2}$ de Pierre Richelet (désormais RICH.) publié en 1680, du Dictionnaire Universel ${ }^{2}$ d'Antoine Furetière (FUR.) paru en 1690, du Dictionnaire de l'Academie ${ }^{2}$ (ACAD.) publié en 1694 et de son complément «technique» le Dictionnaire des Arts et des Sciences ${ }^{2}$ de Thomas Corneille (COR.) publié en cette même année. S'y ajoutent leurs rééditions allant jusqu'en 17013, moment où le vocabulaire de la danse peut être considéré comme formé, consacré par la publication de la Choregraphie, ouvrage fondamental à l'intérieur duquel ce vocabulaire se présente comme un ensemble organisé et homogène.

\section{LA LEXICOGRAPHIE AU XVII' SIÈCLE ET LES VOCABULAIRES TECHNIQUES}

Avant d'examiner la part du vocabulaire de la danse dans ces dictionnaires, il convient d'évoquer la place que la lexicographie de l'époque a accordée aux vocabulaires techniques en général. Cachés dans des traités en latin réservés aux érudits ou cantonnés dans des glossaires à la fin des manuels spéciaux, ceux-ci commencent dès 1675 à faire l'objet de productions spécialisées autonomes, avant d'être recueillis, répertoriés et diffusés systématiquement à partir du milieu du XVIII ${ }^{\mathrm{e}}$ siècle. Mais si le siècle des Lumières a permis la consécration des vocabulaires des arts, des sciences et des métiers, notamment avec l'Encyclopédie de Diderot, celle-ci avait été préparée par quelques dictionnaristes du siècle précédent. En effet, les dictionnaires généraux des $\mathrm{XVI}^{\mathrm{e}}$ et $\mathrm{XVII}{ }^{\mathrm{e}}$ siècles obéissaient à deux tendances. La première privilégiait ce que Quemada (1978: 1156) ${ }^{4}$ appelle leur «rôle de pédagogues» et cette fonction fut assumée par l'ACAD. et par RICH. La seconde en revanche, mettait en avant leur «rôle d'archivistes» du lexique. Le Thresor ${ }^{5}$, comme son titre l'indique, et plus encore le Dictionnaire Universel, relèvent de cette fonction. Ceux-ci sont extensifs, ceux-là sélectifs. Les auteurs des ouvrages dits universels, tel FUR., 
puiseront largement dans les répertoires spécialisés mentionnés ci-avant. Les autres écarteront délibérément les vocabulaires techniques.

Dès les premières pages de la préface de son dictionnaire, l'ACAD. annonce qu'elle «se retranche à la Langue Commune telle qu'elle est dans le commerce ordinaire des honnestes gens et telle que les Orateurs et les Poëtes l'employent», excluant du même coup aussi bien les «termes des Arts et des Sciences qui entrent rarement dans le Discours» que les «vieux mots qui sont hors d'usage» (préf. : XIV). En matière de nomenclature le dictionnaire sera par conséquent sélectif et synchronique. Quant aux normes - sociale et grammaticale - elles sont représentées par le bel usage et le bon usage, l'Académie se réservant le droit d'étiqueter ce qui lui paraît être du «mauvais usage» ou du «style familier». Et voilà sa troisième caractéristique qui est d'être un ouvrage normatif et prescriptif.

Le Dictionnaire François de son côté annonce une nomenclature plus étendue, contenant «tous les mots» et «expressions» aussi bien «propres, figurés que burlesques», avec, en plus, «les termes les plus connus des Arts et des Sciences/le tout tiré de l'usage et des bons auteurs». Ce programme fut respecté dans son ensemble - encore qu'il faille attendre les éditions de 16936 et même de 16946 pour le voir tenir effectivement ses promesses. Cependant, dès $1680, \mathrm{RICH}$. enregistre «de nombreux termes techniques ou archaïques, des vulgarismes et des néologismes» (Quemada 1967:209)7. S'il a dépouillé les «bons auteurs», selon ses propres dires, il semble également avoir rencontré des spécialistes et travaillé de première main comme l'affirment Baldinger $(1951: 346)^{8}$ et Bray (1986: 8)9. Par ailleurs, il prend soin de consigner les différents niveaux et registres de langue, non toutefois sans les caractériser et leur assigner un rang. De toutes ces considérations, nous pouvons déduire que nous sommes en présence d'un dictionnaire du bon usage, normatif mais non puriste, sélectif mais non restrictif.

Tout autre est l'attitude de FUR. qui fera de son ouvrage, bien qu'il s'agisse d'un dictionnaire de mots et non pas de choses, un prototype des dictionnaires encyclopédiques. Le programe que le titre annonce est vaste. Il en résultera un ouvrage universel, pas normatif du tout - les marques de niveaux de langue sont pratiquement absentes, à quelques exceptions près - cumulatif, et dont la richesse ne va pas sans danger pour celui qui le consulte. Représentant la somme du savoir depuis la Renaissance et, au plus tard, jusqu'en 1688 - année de la mort de l'auteur -, le Dictionnaire Universel est un ouvrage de compilation où synchronie et diachronie coexistent sans qu'il y ait toujours une explication ou une mise en garde.

\section{COMPARAISON DES TROIS DICTIONNAIRES}

\section{La nomenclature relative à la danse}

\section{Le Dictionnaire François de Pierre Richelet}

Des dix éditions du dictionnaire de RICH. qui ont paru entre 1680 et 1701 , nous avons pu consulter les cinq dont les exemplaires se trouvent dans les bibliothèques parisiennes, notamment à la Bibliothèque nationale. Parmi elles, celles de 1685 et de 1694 (édit. Dentand) sont des reprises de l'édition originale de 1680 . Si les sept premières n'ont guère apporté de changements quant à la nomenclature, avec celle de Ritter (1693) nous assistons à un profond remaniement à la fois du nombre et du contenu des articles avec parfois mention de l'étymologie ou de la prononciation, assorti d'environ deux cents pages d'une partie intitulée «Augmentation». Celle-ci n'est toutefois pas attribuée à RICH. mais à un «fidèle copiste de $\mathrm{Mr}$ Furetière». La dénonciation venant de Dentand, responsable de l'édition de 1694, paraît vraisemblable à en juger par le remaniement de l'article Pas de Ballet dans l'édition de 1693 qui présente quelques similitudes avec le même article chez FUR. Quant aux autres éditions qui suivront, celle de Dentand reprendra la nomenclature de l'original de 1680 enrichie également d'une partie «Augmentation» qui, en réalité, 
reproduit les articles ayant constitué la nouveauté de 1693 mais qui cette fois est, selon l'éditeur, de la main même de RICH. Enfin, l'édition Gaillard (1694) suivie de Gaillard (1695) strictement identique à la précédente, revient à la nomenclature de Ritter (1693).

Le Dictionnaire François de 1680 enregistre 30 entrées donnant lieu à des développements assez succincts à l'intérieur desquels on dénombre 13 items cachés. Ce qui fait en tout 43 vocables dont deux, pavane et tricotet, déjà vieillis et non usités à l'époque.

\begin{tabular}{|c|c|}
\hline RICHELET 1680 & Maître de danse \\
\hline Bal & Menuet \\
\hline Baladine & Pas \\
\hline Ballet & pas figuré \\
\hline danse figurée & Pavane \\
\hline danse par haut & Pirouetter \\
\hline Branle & Temps \\
\hline $\begin{array}{l}\text { branle gai } \\
\text { maître à danser }\end{array}$ & Tricotet \\
\hline Battre & RICHELET 1693 \\
\hline Bourrée & Battement \\
\hline Cabriole & battement croisé \\
\hline Cabrioler & battement serré \\
\hline Cadence & Entrechat \\
\hline danser en Cadence & cabriole croisée \\
\hline Contretemps & cabriole en avant \\
\hline Coupé & en tournant \\
\hline Couper & de côté \\
\hline Courante & Mouvement \\
\hline Danse & Pas de ballet \\
\hline danse élevée & pas droit \\
\hline danse basse & grave \\
\hline danse figurée & ouvert \\
\hline Danser & battu \\
\hline Danseur & tourné \\
\hline danseur de l'Opéra & tortillé \\
\hline Danseuse & relevé \\
\hline Entrechat & balancé \\
\hline saut figuré & coupé \\
\hline Entrée & dérobé \\
\hline entrée de ballet & glissé \\
\hline Figure & tombé \\
\hline Fleuret & pas mignardés (vieilli) \\
\hline pas de bourrée & pas de danse \\
\hline Gaillarde & les cinq pas (vieilli) \\
\hline
\end{tabular}

Comme on peut le constater, l'édition de 1693 ajoute 25 termes techniques (dont deux vieillis) qui se présentent sous forme soit d'items cachés soit d'éléments définitoires ou même d'exemples : $c f$. ENTRECHAT: «sorte de saut figuré. Ce mot est corrompu de l'italien capriola intreciata. C'est une capriole croisée. (Il y a, un entrechat en tournant, un entrechat en avant, et un entrechat de côté. Un entrechat bien fait)». Ils témoignent du remaniement des articles mentionné plus haut. Remaniement d'ailleurs que pourrait avoir provoqué la parution, entre temps, du Dictionnaire Universel, puisque dans l'édition de $1680 \mathrm{RICH}$. se contente de dire qu'entrechat est une «sorte de saut figuré», donne comme exemple «faire un entrechat» et ne mentionne aucune variante donnant lieu à une dénomination technique. 
Le commentaire en revanche qu'il fait à propos de cabriole est très intéressant. En effet, en pleine période de «naturalisation» du terme, RICH., dans le chapitre des «Remarques sur la lettre C» (1680), fait la distinction entre une forme - capriole appartenant à l'usage technique encore marqué par les origines italiennes du vocabulaire de la danse et une forme banalisée et francisée - cabriole - qui finira par prévaloir: «J'ai dit que cabriole et cabrioler étoient plus usitez que capriole et caprioler. Je ne me puis encore retracter. Capriole et caprioler venant de l'Italien capriola semblent les plus réguliers. Plusieurs personnes qui traitent des arts écrivent et prononcent capriole et caprioler. Mais tout le reste du monde qui parle bien, écrit et prononce cabriole et cabrioler. Il est libre là-dessus de suivre son inclination.» Lorsque l'information livresque ne lui suffit plus, RICH. n'hésite pas à faire une observation in situ, justifiant ainsi la qualification «ouvrage de première main» attribuée à son dictionnaire. Le passage ci-après, tiré de l'édition de 1693, en est la preuve: «(...) Presque tous les danseurs que j'ai vus sur ce mot disent cabrioler.» Plus intéressante encore, la remarque qui suit montre le lien entre fréquence d'usage et banalisation: «(...) cabriole paraît plus usité dans la bouche de ceux qui dansent et qui en font tous les jours.» Enfin, le transfert de battement, du corps de l'article où il figurait en 1693, à la partie «Augmentation» de l'édition de 1694, témoigne de la reprise par Dentand des éléments nouveaux parus dans l'édition de Ritter (1693). D'autres ressemblances frappantes entre ces deux éditions nous font pencher en faveur de l'hypothèse avancée par Bray (1686: 97) selon laquelle RICH. aurait effectivement participé à la révision de 1693 contrairement aux affirmations de Dentand.

\section{Les dictionnaires de l'Académie française}

a) Le Dictionnaire de l'Académie

Pour avoir exclu les vocables techniques de son dictionnaire, l'Académie n'en a pas moins enregistré 62 lexies appartenant au vocabulaire de la danse, dont 33 se présentent comme des vedettes ou des sous-vedettes, tandis que 29 paraissent sous forme d'items cachés. Il est même intéressant de remarquer que cette nomenclature est légèrement supérieure à celle de RICH., ce qui tendrait à prouver que le public auquel l'Académie s'adressait était celui-là même qui avait fait de la danse une de ses occupations préférées, et pour lequel ce vocabulaire, étant devenu «fort commun»selon les propres termes du rédacteur de la préface (1694: XV), avait tout naturellement droit de cité.

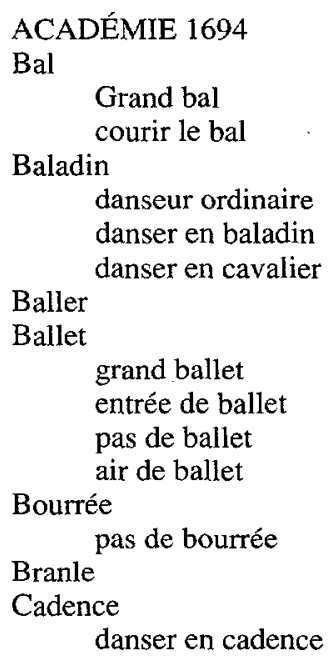

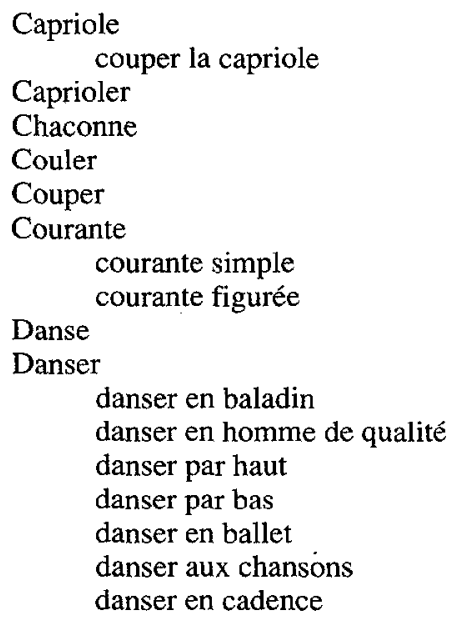



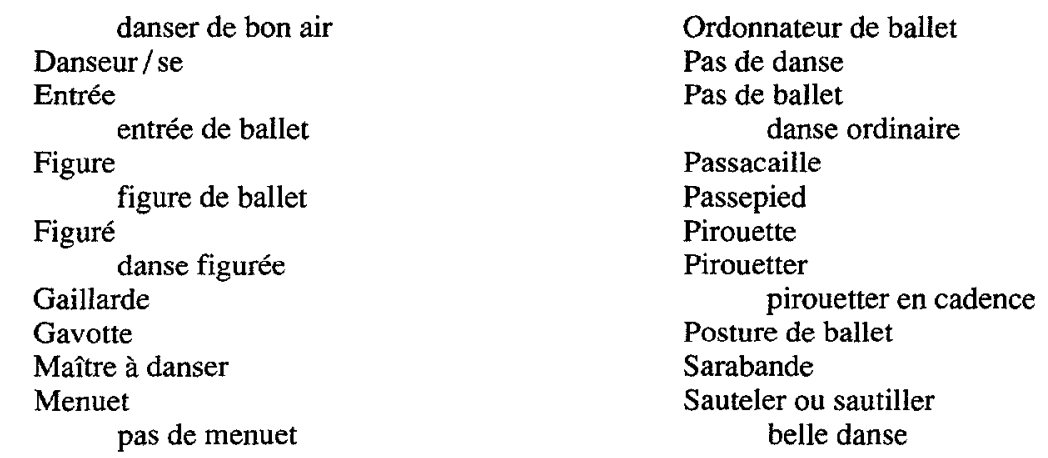

b) Le Dictionnaire des Arts et des Sciences de Thomas Corneille

Usuel et actuel, le vocabulaire présenté ci-avant s'inscrit dans la perspective synchronique annoncée par les auteurs du Dictionnaire de l'Academie. Il n'en va pas de même pour l'inventaire dressé par Thomas Corneille dans le Dictionnaire des Arts et des Sciences. On y trouve quantité de mots appartenant à des pratiques chorégraphiques déjà en désuétude ou en voie de disparition, dont la plupart appartiennent à la danse de la Renaissance et que FUR. avait enregistrées déjà en 1690 dans son Dictionnaire Universel. La similitude même des définitions proposées par les deux ouvrages, fait penser à un plagiat, ce qui n'est pas impensable si l'on tient compte de ce que Bray (1986:8) appelle «la criminalité dictionnairique»10.

CORNEILLE 1694
Coupé
Couper
Courante
pas
balancement
coupé
courante simple
courante figurée
Danse
pas mesurés
danse par haut
gambades
caprioles
Baladins
danse par bas
Danseur
Entretaille
Fleuret
pas de bourrée
Gaillarde
cabrioler
danser terre à terre
cinq pas
assiette de pied
Gavotte
bransle double
Menuet
pas relevé
Pas $\quad$

pas de balet
pas figuré
balet
pas de courante
pas de menuet
pas de gavotte
pas de bransle
pas de Canarie
pas de bocane
pas droit
pas simple
pas grave
pas ouvert
pieds
grand bal
position régulière
friser la cabriole
saut simple
saut battu
saut majeur
Sarabande
s


Des 58 lexies retenues par COR., 8 appartiennent à la danse de la Renaissance qui n'est plus en usage à la fin du XVII ${ }^{\mathrm{e}}$ siècle (entretaile, pavane, pas de bocane, passemeze, ruade, rut de vache, saut majeur, tricotet) tandis qu'une seulement (gaillarde) est qualifiée par le lexicographe d'ancienne. Les deux dictionnaires de 1'Académie totalisent 1.21 vocables dont 17 en double, ce qui fait 104 lexies en tout. Entre le répertoire banalisé et synchronique du dictionnaire proprement dit et celui, un peu plus technique, du supplément consacré aux arts et aux sciences, l'Académie couvre honorablement ce domaine spécialisé.

\section{Le Dictionnaire Universel d'Antoine Furetière}

La nomenclature relative au vocabulaire de la danse relevée dans l'ouvrage de Furetière surprend non seulement par son étendue mais aussi par le fait qu'elle pose le problème de ses sources. Il est vrai que sur ce dernier point on est en droit de se poser plusieurs questions. Quand et par quel moyen FUR. est-il entré en possession des renseignements hautement techniques qu'il nous livre dans son dictionnaire? A-t-il eu des contacts avec les professionnels? A-t-il utilisé des manuels spécialisés? Et si oui, lesquels ? Dans l'édition critique du Dictionnaire Universel, parue en 1978, Alain Rey ${ }^{11}$ donne la liste des ouvrages qui, selon lui, auraient servi à Furetière de corpus pour la danse. Il s'agit de La Muze historique de Loret, des Mémoires de Marolles, des Historiettes de Tallement des Réaux, de l'Orchesographie de Thoinot Arbeau, de l'Harmonie Universelle de Mersenne. Parmi eux, les deux derniers ainsi que le traité, capital, de Menestrier, sont mentionnés par l'auteur lui-même. Cependant, pour avoir examiné très attentivement ces ouvrages, nous sommes persuadée que ce ne sont pas eux qui ont permis à FUR. d'être à la fois si exhaustif et si actuel. Car ce qui est impressionnant pour le chercheur en danse d'aujourd'hui, c'est de voir consignée dans un dictionnaire, qui, comme c'est bien connu, est la somme de ce qui a été plutôt que de ce qui est au moment de sa parution, la totalité d'un vocabulaire qui n'obtiendra sa consécration que dix ans plus tard. On peut nous rétorquer, à juste titre, que le dictionnaire n'a fait qu'entériner un usage oral répandu dans les milieux concernés. Soit. Mais le traitement par exemple de l'article pas chez FUR., dépassant l'organisation de la matière lexicale proprement dite et nous renseignant de façon égale sur le mot et sur la chose, devient le premier témoignage des recherches entreprises alors sur l'analyse du mouvement. Or, si celles-ci avaient abouti et si des ouvrages avaient paru, FUR. les aurait certainement mentionnés. Ce ne fut apparemment pas le cas. En revanche, l'auteur donne l'impression d'avoir observé les spécialistes (bien qu'à aucun moment il ne le confirme comme le fait p. ex. RICH.) dans les ateliers où cette analyse s'élaborait et il nous donne en primeur le résultat de leurs travaux. En outre, lorsqu'on sait que Furetière mourut en 1688 et que la majeure partie de son corpus fut constituée entre 1650 et $1685^{12}$, on est tenté de situer ces travaux aux alentours de ces dates; ce qui correspond aussi bien aux efforts déployés par Beauchamp ${ }^{13}$ vers 1670 , Lorin ${ }^{13}$ vers 1685 et en 1688 , et Favier ${ }^{13}$ en 1688 pour élaborer un code extralinguistique susceptible de transcrire le mouvement. La fréquentation des ateliers par l'auteur du Dictionnaire Universel se confirme également par la mention qu'il fait de lexies synonymes pour désigner le même mouvement; preuve que Furetière se documenta auprès de différents maîtres à danser dont on sait que chacun usait d'un vocabulaire plus ou moins personnel ou bien donnait une acception particulière à une lexie commune à tous ${ }^{14}$. Quant à l'étendue de la nomenclature, A. Rey, dans l'Index thématique qu'il a établi, avance le nombre de 36 termes de danse. Cette estimation semble approximative. Ne tenant compte apparemment que des vedettes, Rey néglige les nombreuses sous-vedettes et les items cachés qui multiplient par trois, et même au-delà, le nombre de vocables recueillis. On trouvera ci-après la liste de ces termes, au nombre de 116, dont 63 items cachés et 14 appartenant à la danse de la Renaissance. 
FURETIÈRE 1690

Bal

Balancement

Balladin

Baller

Ballet

vers de ballet

Bourrée danse figurée

Bransle

bransles doubles

bransles communs

bransle simple

bransle gay

bransle à mener

bransle de sortie

bransles morgués

Cabriole bransles gesticulés

Cadence

$$
\text { demi-cabriole }
$$

entrer en cadence sortir de cadence

Canarie

Capriole

Caprioler

Contretemps

Couler

Coupper

Courante

courante simple courante figurée couppé

Chaconne

Danse

danse par haut

Danser danse par bas

Danseur/se danseur ordinaire

Entrechat

Entrée entrée de ballet

Entretaille

Fleuret

Figure

Gaillarde

Gavotte

Gigue

Maître à danser

Menuet

Mouvement

Pas

pas droit

grave ouvert

battu

tourné

tortillé

en dehors

en dedans

relevé

balancé

coupé

dérobé

glissé

chassé

tombé

mignardé

pas de Courante

Bourrée

Menuet

Gavotte

Bransle

Canarie

Traquenard

Bocanne

Sissonne

Balet

les cinq pas

demi-coupé

tour de jambes

temps relevé

pas avec mouvement

Passemeze

Passecaille

Passepied

Pavane

Pirouette

demi-pirouette

Pirouetter

Position

la première

la seconde

la troisième

la quatrième

Posture

Redanser

Ruade

(Salle) tenir salle

Sarabande

Saut

saut simple

saut battu

saut de Basque

coupé sauté en tournant

saut majeur

pas sauté

Soubresaut 


\begin{tabular}{ll}
\multicolumn{1}{c}{ souple saut } & Tricotet \\
Temps & (Vache) ru de vache \\
Tordion & Volte \\
Traquenard & Voltiger
\end{tabular}

Comparativement à la nomenclature des autres dictionnaires, on en conviendra, celle de FUR. est sans commune mesure. Là où les autres dictionnaristes enregistrent un vocabulaire largement banalisé depuis un siècle, FUR. fournit au lecteur de l'époque et à plus forte raison au chercheur d'aujourd'hui, le premier répertoire digne de ce nom des termes de danse du XVII ${ }^{\mathrm{e}}$ siècle Certains seront même datés de 1690, n'étant pas encore repérés dans des ouvrages antérieurs au Dictionnaire Universel. Il s'agit des:

Pas battu
dérobé
droit
grave
ouvert
relevé
tortillé
tourné
coupé sauté en tournant
demi-cabriole
demi-couppé

demi-pirouette
saut de Basque
saut simple
temps relevé
tour de jambes
balancement
position
première
seconde
troisième
quatrième

La seconde édition de 1694 n'apporte guère de modifications. Celle de 1701, établie sous la surveillance de Basnage, n'enrichira pas la nomenclature relative à la danse, mais procédera à une «normalisation» des données et surtout «corrigera» à l'intérieur des articles les excès ou les omissions volontaires dus en grande partie aux démêlés de l'auteur aussi bien avec les institutions qu'avec des individus. Ainsi, la mort en 1691 du poète et académicien Benserade, ennemi juré de FUR, à l'Académie, sera l'occasion de compléter l'article BALLET en rappelant sa longue et précieuse collaboration dans le ballet de Cour.

De ce rapide tour d'horizon se dégage la constatation suivante. Le vocabulaire de la danse consigné dans les trois grands dictionnaires monolingues de la fin du XVII ${ }^{\mathrm{e}}$ siècle, concerne les différentes sortes de danses et de ballets, les divers styles, les actions et les pas fondamentaux ainsi que les artisans (danseurs, compositeurs - c'est le mot dont on se sert alors pour parler des chorégraphes - , ordonnateur etc.). Ce vocabulaire, largement banalisé, est immédiatement décodé par le lecteur de l'époque. Mais le vocabulaire technique du mouvement qui implique un «savoir» préalable, ne fait partie que de la nomenclature du FUR. et de celles qui, parmi les rééditions des autres dictionnaires, furent publiées après 1690 et par conséquent largement inspirées de lui.

\section{Le traitement de la matière lexicale : l'organisation des articles; les définitions ; les exemples; les citations}

Les articles

Examinons maintenant la façon dont l'information est traitée. L'accès à cette information se fait par l'intermédiaire du mot vedette. Mise à part la première édition du dictionnaire de l'Académie qui suit un classement par racines, tous les autres ouvrages de notre corpus classent les vedettes dans l'ordre alphabétique.

Appartenant à un vocabulaire spécialisé, le terme de danse est souvent placé en sous-vedette, précédé de la mention «en termes de Danse» (FUR.) ou «terme de Maître de Dance» (RICH.). L'ACAD. utilise le plus souvent comme entrée en matière l'expression «on dit...» ce qui met en évidence le caractère banalisé des termes consignés. Pour être 
facilement repérable, le nouveau sens se voit attribuer une entrée soit en saillie (RICH.) soit en retrait (ACAD.). L'organisation interne des articles privilégie d'abord le sens propre illustré par des exemples ou par des citations selon les prises de position de chaque dictionnaire et accompagné des éléments linguistiques fondamentaux pour la compréhension du mot tels que les épithètes et les collocations les plus usuelles. Vient ensuite le sens figuré. C'est à cet endroit que l'ACAD. place un nombre considérable de proverbes, option qui lui fut beaucoup reprochée mais qui s'avère d'un grand secours pour le chercheur en danse qui peut à travers eux constater l'impact de la danse auprès du public mais aussi suivre parfois son évolution 15 . Il est intéressant de remarquer à ce propos, que le sens figuré «faire danser quelqu'un» (= se moquer, berner) fut par la suite récupéré par le verbe marcher. Serait-ce le fait d'une société moins «dansante»?

\section{Les définitions}

Elles commencent à se développer avec les dictionnaires monolingues. RICH. puis FUR. et enfin l'ACAD. fixèrent, les premiers, les procédés définitoires qui sont encore en usage aujourd'hui. La plupart des définitions du vocabulaire de la danse, tous dictionnaires confondus, sont des définitions en extension. La lexie à définir est décrite à l'aide d'un ou plusieurs traits spécifiques. RICH. p. ex. définit ainsi le branle: «Dance où plusieurs dancent en rond, se tenant par la main.» Les deux exemples qu'il donne: «un branle gai, mener un branle», complètent à leur manière la définition puisque les collocations fournies servent à désigner un hyponyme et à renseigner sur une des façons d'exécuter cette danse qui, d'ailleurs, avait donné lieu à la dénomination technique branle à mener. La définition de l'ACAD. n'apporte rien de nouveau. Celle de FUR. en revanche est très complète. L'auteur, en effet, définit d'abord l'hyperonyme en mentionnant les pas utilisés, puis donne la «differentia specifica» des hyponymes spécifiques, l'ordre dans lequel ils doivent être exécutés, la mesure musicale et enfin il cite sa source ; le tout entrecoupé de développements encyclopédiques; $c f$ : «BRANSLE, en termes de Musique, est un air ou une danse par où on commence tous les bals, où plusieurs personnes dansent en rond, (...) en se tenant par la main (...). Les bransles consistent en trois pas et un pied joint (...). Quand ils sont répétés deux fois, ce sont des bransles doubles ou communs. On danse d'abord le bransle simple, et puis le bransle gay par deux mesures ternaires; et il est ainsi appelé, parce qu'on a toûjours un pied en l'air. Voyez Thoinot Arbeau dans son Orchesographie (...).»

Les autres procédés définitoires, tels que l'emploi de substituts nominaux et plus particulièrement de synonymes, ne seront pas beaucoup utilisés car ils ne conviennent pas à l'objet traité. Cependant, dans la mesure où la fin du XVII ${ }^{\mathrm{e}}$ siècle est une période de formation et de mise en place de ce vocabulaire, les lexicographes auraient pu faire appel à eux. C'est chez FUR. que l'on rencontre plusieurs dénominations synonymiques, par exemple fleuret=pas de bourrée, pirouette=tour, saut de Basque=coupé sauté en tournant, entrechat=capriole croisée, etc. Ainsi, on peut suivre presque pas à pas l'élaboration de ce vocabulaire à travers les tâtonnements de la première heure et les réalisations concurrentes avant qu'un large consensus l'emporte en faveur de telle ou telle lexie.

Le traitement de la lexie danseur chez RICH.(1680) mérite d'être souligné. En proposant deux entrées distinctes pour danseur et danseuse, l'auteur, en bon «sociologue du langage», (Bray 1986:38) traduit une réalité sociale relative au statut des femmes dans la profession. En effet, la suprématie des hommes, danseurs professionnels ${ }^{16}$, est à cette époque unanimement reconnue. On rejoint alors les considérations de Benveniste ${ }^{17}$, puisque la définition de danseur chez RICH. met l'accent sur le trait spécifique «métier» tandis que celle de danseuse ressemble plus à ce que Benveniste appelle une description; $c f$. DANSEUR «celui qui dance. celui qui fait metier de dancer. (C'est un des danseurs de l'Opéra.)»; DANSEUSE «celle qui dance bien. (c'est la meilleure danseuse du monde)». 
La danseuse de RICH., occasionnelle, ne sera définie que par rapport à la qualité de sa danse et non pas par son statut. Lorsque, dix ans plus tard, le nombre de danseuses professionnelles sera pratiquement égal à celui des danseurs, les dictionnaires de FUR. et de l'ACAD. en témoigneront en leur consacrant une seule entrée.

La richesse et la qualité du travail définitionnel de FUR. tentèrent quelques lexicographes moins documentés ou moins inspirés que lui. L'examen attentif des définitions fournies par les trois dictionnaires révèle de nombreux «emprunts» faits par COR. surtout à FUR. 18 mais aussi à RICH. En voici quelques exemples:

\section{FURETIÈRE 1690}

«(...) La Danse par haut, est celle des Baladins, qui font des cabrioles et des gambades. Danse par bas est celle qui se fait modestement et terre à terre, comme celle des honnestes gens.»

\section{CORNEILLE 1694}

«Il ya deux sortes de Danse, l'une appellée Danse par haut, qui consiste dans des gambades et les caprioles que font les Baladins, et l'autre appellée Danse par bas. Celle-là se fait à terre et d'une manière modeste.»

Comme on peut le constater, en reproduisant les articles de Furetière, Corneille a pris soin de dissimuler les extraits «empruntés» soit en inversant l'ordre des mots ( $c f$. Baladins, cabrioles, gambades $\longrightarrow$ gambades, caprioles, Baladins), soit en transformant les éléments articulateurs (qui font des... $\rightarrow$ qui consiste dans...). Parfois, il lui arrive même de reprendre la définition presque mot pour mot, ce qui est le cas de l'article SAUT :

\section{FURETIÈRE 1690}

«Se dit d'un pas de balet, des danses par haut, où on élève en même temps son corps et ses deux pieds en l'air pour friser la cabriole: ce qui se fait ordinairement à la fin d'un couplet, et pour marquer les doubles cadences. Le saut simple ou pas sauté, c'est lors que les jambes estant en l'air ne font aucun mouvement, soit qu'il se fasse en avant, en arrière ou de costé. Le saut battu c'est lorsque les jambes estant en l'air, les talons battent l'un contre l'autre une, ou plusieurs fois: et quand on passe les jambes l'une par dessus l'autre par trois fois, cela s'appelle entrechat. Le saut de Basque est un couppé sauté en tournant. On appelle aussi le saut majeur, cabriole, quand on remuë les pieds en l'air et quelques-uns l'appellent cadence. $(. ..) \gg$

\begin{abstract}
CORNEILLE 1694
«(...) On appelle Saut en termes de danse un pas de balet qui se fait en elevant en mesme temps son corps et ses deux pieds en l'air pour friser la cabriole; ce qui se fait d'ordinaire à la fin d'un couplet et pour marquer les doubles cadences. On dit qu'on fait un saut simple, quand les jambes estant en l'air ne font aucun mouvement, soit qu'il se fasse en avant, en arrière ou de costé. Le saut battu, est celuy où les jambes estant en l'air, les talons battent une ou plusieurs fois l'un contre l'autre. On appelle saut majeur celuy où l'on remuë les pieds en l'air.»
\end{abstract}

Notons au passage le choix délibéré d'omettre ce qui dans le développement de l'article paraît soit trop technique, $c f$. coupé sauté en tournant soit étymologiquement difficile à classer $c f$. entrechat. Dans la thèse qu'il a consacrée à l'auteur du Dictionnaire François, Bray (1986: 42) a mis en évidence le fait que COR. s'était «très largement inspiré du travail définitionnel» de RICH. Cependant un examen approfondi révèle qu'en matière de vocabulaire de la danse, COR. a surtout profité des recherches de FUR. tant pour la nomenclature que pour les définitions. D'ailleurs, il n'est pas exclu qu'il ait puisé simultanément dans les deux ouvrages comme semble le suggérer l'article FLEURET:

CORNEILLE 1694

«On appelle aussi Fleuret en termes de Danse, un pas de bourrée qui est une danse fort gaye. Ce pas est composé de trois pas joints ensemble, mais il n'y a qu'un mouvement» 


\section{RICHELET 1680}

«Terme de Dance. C'est un pas de bourrée, qui est une sorte de dance gaie (Vois-tu ce petit trait? ce fleuret? ces coupés? Moliere, Les Fâcheux acte I sc. 3)»

\section{FURETIÈRE 1690}

«est aussi le nom d'un pas qu'on fait à la danse; ou plutost ce sont trois pas joints ensemble, mais il n'y a qu'un mouvement. C'est la Bourrée des Basques.»

Le Dictionnaire Universel à son tour - mais ce n'est bien sûr pas FUR., c'est Basnage qui surveilla l'édition de 1701 - se verra enrichi de quelques ajouts dont certains sont «empruntés» presque mot pour mot au dictionnaire de l'ACAD. ou à celui de RICH. Ainsi, à l'article ENTRECHAT on ajoutera une note sur l'étymologie du mot: «(...) ce mot est corrompu de l'italien capriola intrecciata, qui signifie une capriole croisée. Il y a un entrechat en tournant, un entrechat en avant et un entrechat de côté.» (À rapprocher de la citation infra p. 4, RICH. 1693.) Quant à l'article DANSE, il sera complété par le commentaire suivant qui ressemble étrangement à l'exemple donné par le dictionnaire de l'ACAD.; $c f$. FUR. 1701 : «Se dit aussi quelquefois pour l'action de danser. Il a une danse contrainte. Il y a du plaisir à voir danser ce jeune Seigneur. Sa danse est noble, libre, aisée»; et ACAD. «(...) Danse signifie aussi l'action de danser, Il a une danse contrainte, il a une danse noble, libre, aisée.»

\section{Les exemples; les citations}

Ce sont les phrases d'exemples qui, au XVII' siècle, seront au cour de la problématique lexicographique. On sait que très tôt l'Académie opta pour elles et que ce choix lui valut de nombreuses critiques de la part des partisans des citations. RICH. et FUR. de leur côté, ne dédaignèrent pas de citer chaque fois que cela était possible. Nous n'entrerons pas dans les détails de cette polémique qui dépasse le cadre de cet article. Mais il est vrai qu'en matière de vocabulaire de la danse ces prises de position n'apparaissent pas très clairement car, les écrits étant rares, les citations le devenaient aussi. Et même losqu'on en trouvait, leur contenu informationnel était réduit. Cependant, les deux citations de RICH. tirées de l'œuvre de Molière, la première des Fâcheux (1661) et la seconde du Bourgeois Gentilhomme (1670), permettent de mesurer l'importance de telle ou telle danse dans le contexte de l'époque. Car ce n'est pas un hasard si Molière, observateur attentif des mœurs de son temps, fait faire à Lisandre, en 1661, «certain air de courante», alors que neuf ans plus tard il fait apprendre le menuet à Monsieur Jourdain 19. Les exemples, au contraire, avaient un rôle à jouer: fonctionnels, ils pouvaient éclairer la définition, parfois la compléter, voire donner des indications de style : $c f$. ACAD. 1694: «Sauteler ou sautiller, sauter à petits sauts (...) ce danseur ne fait que sautiller, dans la belle danse, il ne faut point sautiller», et à propos de MASCARADE: «Divertissement, danse, momerie de gens qui sont en masque. ce fut une plaisante mascarade. ce n'est pas un ballet; c'est seulement une petite mascarade.». Les collocations aussi pouvaient être précieuses. C'est encore le dictionnaire de l'ACAD. qui est le plus riche comme peut en témoigner l'article DANSE: «(...) danser bien, danser mal, danser en baladin, danser en homme de qualité. danser de bon air. danser par haut, danser par bas. danser en ballet. danser en chansons. danser en cadence. danser sur la corde. montrer à danser. apprendre à danser. maistre à danser. On danse chez un Tel, c'est à dire il y a bal etc.» FUR. ne semble pas attacher beaucoup d'importance à ces points mais c'est uniquement parce que les collocations les plus importantes, celles qui étaient déjà lexicalisées ou en voie de lexicalisation, faisaient partie du corps de l'article et très souvent avaient leur propre définition.

\section{FURETIÈRE AU SECOURS DES CHORÉLLOGUES}

Des considérations qui précèdent, il résulte que ce domaine d'expérience est bien représenté dans les trois grands dictionnaires monolingues de la fin du XVII ${ }^{\mathrm{e}}$ siècle et ce, 
quelle qu'ait été la prise de position lexicographique des auteurs. Procédant, chacun, à une approche différente de cet art, ils finissent par donner à eux trois une image fidèle et complète de la danse à cette époque. Mais d'où vient-il alors que FUR., en dehors de l'intérêt qu'il représente pour le linguiste, exerce une véritable fascination sur les choréologues de notre époque? Quelle est l'originalité de son dictionnaire par rapport aux autres?

Certes, l'originalité de FUR. réside dans son travail définitionnel qui découle de son intention de faire un dictionnaire des arts et des sciences, donc de traiter aussi bien les mots que les choses. Ses définitions des pas de danse sont non seulement complètes mais... dansantes ! Elles décrivent les mouvements dans les moindres détails et, parce que FUR. n'était que lexicographe et non danseur, il décrivit même ce qui paraissait évident chez les professionnels et que les ouvrages spécialisés parus à l'aube du XVIII ${ }^{\mathrm{e}}$ siècle, telle la Choregraphie, ne mentionneront pas. Ce fut le cas de la lexie mouvement qui, en dehors de son acception habituelle, désignait ce qu'en danse nous appelons communément plié: cf. FUR. 1690 «On appelle les pas avec mouvement ceux qui se font avec plis des genoux.». Du coup, les lexies pas de bourrée, coupé à deux mouvements deviennent claires pour le choréologue d'aujourd'hui qui, loin de la compétence de l'artiste du XVII siècle, n'a que son imagination et son intuition pour suppléer au manque d'information 20 . C'est d'ailleurs à FUR. que nous devons la description en langue du pas de bourrée à deux mouvements avant 1725 ; $c f$. BOURRÉE: «(...) est aussi une espèce de danse composée de trois pas joints ensemble par deux mouvements (...) Elle est composée d'un balancement et d'un coupé.»

Ce qui est unique chez FUR., c'est d'avoir presque systématiquement accompagné la définition d'une danse de celle du pas qui lui est propre, comme on vient de le voir pour la bourrée et pour le branle (v. infra, p. 12). Par ailleurs, sa description de la courante permet - par recoupement bien sûr avec d'autres sources ${ }^{21}$ — de reconstituer le pas de courante avec ses deux variantes : le pas long et le pas court, qui ne seront consignées dans aucun traité français et pour cause. La courante n'est plus en vogue à la fin du siècle puisqu'elle fut supplantée par le menuet autour de 1670. Et que dire de ses définitions des verbes qui désignent des actions en danse et qui nous donnent les clés du style? cf. COULER «en termes de danse, signifie porter sa jambe doucement, legerement et à fleur de terre. La danse consiste à sçavoir bien couler, couper et piroüetter.».

Un autre apport considérable du dictionnaire de FUR. est qu'il donne tous les synonymes d'une lexie, ce qui permet de suivre le travail de codification du vocabulaire de la danse à mesure que les recherches sur l'analyse du mouvement obligeaient les notateurs à opérer des choix de dénomination et à redistribuer le vocabulaire. La lexie demi-coupé est à ce titre intéressante. C'est FUR. qui est, très vraisemblablement, le premier à la consigner en établissant d'ailleurs la synonymie avec balancement ou pas balancé. Or, ces derniers existaient déjà dans les deux ouvrages de Lorin (V. note 12) en 1685 et 1688 et selon toute vraisemblance remplissaient les fonctions du demi-coupé. La dénomination demicoupé, enregistrée par FUR. en 1690, correspond grosso modo au moment de l'éclosion de l'écriture du mouvement qui a nécessité une analyse rigoureuse des pas. Or, c'est peutêtre en analysant le pas balancé que l'on s'aperçut qu'il constituait la moitié d'un coupé22. Il se peut que cette dénomination ait été créée pour obéir au besoin qui se faisait sentir à l'époque de construire un vocabulaire cohérent conforme aux principes de rigueur, de clarté et de justesse, entre autres, si caractéristiques du classicisme français. Toujours est-il qu'en 1700 Feuillet consacre définitivement l'usage du demi-coupé mais passe sous silence le pas balancé qui, faisant double emploi, manifestement, n'avait pas de place dans le système ainsi créé. Il ne restait plus au pas balancé que cette alternative : subir une mutation de contenu ou disparaître. C'est le premier qui se produisit. En 1725, lors de la redistribution du vocabulaire, il eut sa nouvelle place et la maintient jusqu'à ce jour ${ }^{23}$. 
Ainsi, l'année 1700 constitue une étape dans le vocabulaire de la danse. Étape dont on saisit d'autant mieux l'importance que FUR. avait permis, dix ans plus tôt, d'en suivre le chemin et de découvrir le stade intermédiaire. Pour le choréologue, le Dictionnaire Universel constitue un trésor inestimable tant pour la richesse de sa nomenclature que pour la précision et la finesse de ses descriptions. L'ampleur de ses développements encyclopédiques aide à replacer les danses dans leur contexte socio-historique. À notre époque de fureur du baroque, où l'on découvre avec délice les réalisations lyriques $d u$ Grand Siècle, Furetière nous assiste et nous guide et c'est une bien grande chance. En 1700 lors du Bal en Masque, et plus encore en 1708, on ne put danser le branle à mener par lequel ordinairement tout bal commençait, car personne ne s'en souvenait, même pas les maîtres à danser24! Mais si le branle avait été encore en usage, c'est sûr que Furetière l'aurait mené...

\section{Notes}

1. Choregraphie ou l'art de décrire la dance par caractères, figures et signes demonstratifs avec lesquéls on apprend facilement de soy-mesme toutes sortes de dances, ouvrage très utile aix maîtres à dancer, Paris chez Brunet 1700. C'est le premier livre qui procède à une analyse du mouvement qui ne fait pas appel la langue. Il connut un énorme succès plébicité par un article dithyrambique paru dans le Mercure galant en cette même année. Il fut aussitôt traduit en anglais. Les Italiens, les Espagnols et les Allemands firent des traductions-adaptations.

2. Richelet, Pierre, Dictionnaire françois contenant les mots et les choses, plusieurs nouvelles remarques sur la langue françoise (...). Avec les termes les plus connus des arts et des sciences, Genève, J. h. Widerhold, 1680. Réditions : Dictionnaire françois contenant generalement tous les mots et plusieurs remarques sur la langue françoise..., Genève, D. Ritter, 1693

Dictionnaire françois de P. Richelet..., Genève, J. J. Dentand, 1694

Furetière, Antoine, Dictionnaire Universel contenant generalement tous les mots français tant vieux que modernes et les termes de toutes les Sciences et des Arts... La Haye et Rotterdam chez A. et R. Leers, 1690. Rééditions : 1694 id.; 1701 deuxième édition Revue, corrigée et augmentée

Dictionnaire de l'Academie françoise À Paris chez Jean-Baptiste Coignard, 1694

Corneille, Thomas, Dictionnaire des Arts et des Sciences, Paris, 1694.

3. En 1701 parut la seconde édition de la Choregraphie qui «répare» les oublis et omissions involontaires de la première. On ne peut donc consulter l'une sans l'autre.

4. Quemada, Bernard, «Technique et langage», in Histoire des Techniques, B. Gille, Paris, Gallimard, Encyclopédie de la Pléiade, 1978, pp. 1146-1240.

5. Nicot, Jean, Thresor de la langue françoyse tant ancienne que moderne..., Paris, D. Douceur, 1606.

6. Pour les rééditions $V$. note 2.

7. Quemada, Bernard, Les dictionnaires du français moderne 1539-1863. Étude sur leur histoire, leurs types et leurs méthodes, Paris, 1967.

8. Baldinger, Kurt, «Autour du "Französisches Etymologisches Wörterbuch" (FEW). Considérations critiques sur les dictionnaires français, Aalma 1380 - Larousse 1949», in Revista Portuguesa de Filologia, IV /2, 1951, pp. 342-373.

9. Bray, Laurent, César-Pierre Richelet (1626-1698). Biographie et auvre lexicographique, Tübingen, Max Niemeyer Verlag, 1986.

10. Idem. Pour la comparaison des dictionnaires de Furetière, Corneille et Richelet V. pp. 12-13.

11. Rey, Alain, «Structures d'un dictionnaire», article paru dans la réimpression en 1978 du Dictionnaire Universel de Furetière SNL - Le Robert.

12. Ceci peut être corroboré par le fait suivant: dans le Dictionnaire Universel figure l'article «Orchesographie» où l'on peut lire: «Art et description de la danse, dont les pas sont notés avec les nottes (sic) de Musique. Il y a un Traité curieux fait par Thoinet (sic) Arbeau imprimé à Langres en 1588 qu'il a intitulé Orchesographie; c'est le premier ou peut-être le seul qui a noté et figuré les pas de la danse de son temps, de la même manière qu'on note le chant et les airs.» Or, si Furetière mentionne ce traité, c'est peut-être que les spécialistes étaient en train de travailler sur l'écriture du mouvement et qu'ils avaient à cet effet exhumé les vieux traités et les vieilles méthodes - car il y en avait d'autres - pour faire le point ou pour s'en inspirer.

13. Vers 1660 Louis XIV avait chargé Beauchamp qui était son maître à danser d'élaborer une méthode d'écriture de la danse ; ce qu'il fit, d'après les témoignages de ses contemporains. La paternité de cette méthode, aujourd'hui perdue, fut à l'origine d'un procès retentissant entre Lorin et Beauchamp. Vers 1686 Lorin présente au roi un manuscrit contenant une méthode de notation des contredanses : Livre de Contredance 
presenté au Roy par André Lorin academicien de sa Majesté pour la Danse. En 1688 il présente un second manuscrit : Livre de la Contredance du Roy. Sa méthode qui fait appel au dessin, aux symboles - par l'utilisation des lettres de l'alphabet -, et aux explications en langue, n'eut pas de succès. La récente découverte du manuscrit de Favier, Le mariage de la grosse Cathos, jette un éclairage nouveau sur l'écriture de la danse mais ne présente pas d'intérêt pour le linguiste puisque les pas ne sont pas nommés.

14. C'est pourquoi dans la préface de la Choregraphie Feuillet précise bien que $\mathrm{s}^{\prime} \mathrm{il}$ explique les «termes affectés à la Dance» alors qu'ils «portent avec eux une explication assez claire», c'est pour «empêcher qu'on n'attache d'autres idées aux mots». La normalisation du vocabulaire de la danse constituait d'ailleurs une des tâches que s'était assignée l'Académie Royale de Danse. Mais aucun écrit n'arriva jusqu'à nous.

15. Par exemple, l'importance du branle durant le XVI ${ }^{e}$ siècle nous est révélée par l'expression : «il ne sçait de quel costé branler» (ne savoir que faire, hésiter) tirée de l'édition de 1549 du Dictionnaire François-Latin de R. Estienne et reprise par Nicot (1584 et 1606). Son remplacement par : «il ne sait sur quel pied danser» (Cotgrave 1611) peut faire penser qu'on avait conceptualisé l'expression en passant du spécifique branler au générique danser. Il préfigure l'importance de plus en plus grande que cet art, qui n'est alors qu'une activité sociale, revêtira au cours du siècle.

16. Depuis le Balet Comique de la Royne, représenté en 1581 au Louvre, et pendant un siècle les hommes régnèrent sur la scène. La profession de danseuse demeure marginalisée jusqu'en 1680 environ. Dans les bals, en revanche, les femmes pouvaient déployer tous leurs talents. À ce propos, on peut faire remarquer que la grâce et la virtuosité de Mademoiselle de Blois, de la princesse de Conti et des Duchesses de Bourgogne et du Maine demeurèrent légendaires.

17. Benveniste, Émile, Problèmes de Linguistique générale, Paris, Gallimard, 1966-1967 et réédition 1974 (celle qui est citée) pp. 116-117.

18. Ceci est valable pour le vocabulaire de la danse car, d'une manière générale, Corneille s'est surtout «inspiré» du travail définitionnel de Richelet; $c f$. Bray, op. cit., p. 42.

19. Molière, Jean-Baptiste Poquelin, Les Fâcheux, 1661, acte I scène V; et Le Bourgeois Gentilhomme, 1670, acte I scène II.

20. Entendre par là l'information de première main, contemporaine des événements. Car depuis la publication de la Choregraphie et durant tout le XVIII ${ }^{e}$ siècle nous possédons une documentation substantielle.

21. Notamment l'ouvrage monumental de Gottfried Taubert, Rechtschaffener Tantzmeister... Leipzig, Friedrich Lanckischens Erben, 1717. Ayant étudié la danse auprès des maîtres les plus renommés de Paris vers le milieu du XVII ${ }^{e}$ siècle, l'auteur nous fait découvrir toute l'activité chorégraphique de cette époque dont nous possédons peu de renseignements, essentiellement sur la technique.

22. Le procédé se systématisera par la suite. La demi-cabriole et la demi-pirouette figurent déjà en 1690 chez FUR. Le demi-entrechat et la demi-position datent de 1700. Pierre Rameau dans son Maître à Dancer proposera les demy-jeté, demy-balancé et demy-contretemps. Plus près de nous on crééra les lexies demi-plié, demi-hauteur, etc. Toutes, sans exception, désignent la moitié du pas qu'elles déterminent.

23. Pour plus de renseignements sur le vocabulaire de la danse, V. Eugénia Kougioumtzoglou-Roucher, Aux origines de la danse classique. le vocabulaire de la Belle Dance : 1661-1701, Thèse Nouveau régime sous la direction de Bernard Quemada, Paris XIII, 1990.

24. Dans le Règlement pour le Bal en masque du 27 Janvier 1700, on lit: «Le Bal commença sans Branle.» Ce qui pouvait n'être qu'un incident de parcours, se confirme par le Marquis de Sourches qui note dans son journal le 8 janvier 1708: «Le Roi avoit dit qu'il vouloit qu'on rétablît l'ancienne coutume de commencer les bals par le branle à mener et par les courantes, mais elle avoit étê si bien abolie que les maîtres de danse qui se trouvèrent à Versailles ne purent pas même montrer le branle à mener aux personnes qui voulurent l'apprendre; et ainsi, on fut réduit à commencer le bal par le menuet.» cf. Memoires du marquis de Sourches sur le règne de Louis XIV (1681-1712), Paris, Hachette 1882-1893. 